\title{
FUNDAMENTAL INVESTIGATIONS OF AIRFRAME NOISE ${ }^{1}$
}

\author{
M.G. Macaraeg2 \\ NASA Langley Research Center \\ Hampton, VA, USA
}

\begin{abstract}
$\underline{\text { Abstract }}$
An extensive numerical and experimental study of airframe noise mechanisms associated with a subsonic high-lift system has been performed at NASA Langley Research Center (LaRC). Investigations involving both steady and unsteady computations and experiments on a small-scale, part-span flap model are presented. Both surface (steady and unsteady pressure measurements, hot films, oil flows, pressure sensitive paint) and offsurface (5 hole-probe, particle-imaged velocimetry, laser velocimetry, laser light sheet measurements) were taken in the LaRC Quiet Flow Facility (QFF) and several hard-wall tunnels up to flight Reynolds number. Successful microphone array measurements were also taken providing both acoustic source maps on the model, and quantitative spectra. Critical directivity measurements were obtained in the QFF. NASA Langley unstructured and structured ReynoldsAveraged Navier-Stokes codes modeled the flap geometries excellent comparisons with surface and offsurface experimental data were obtained.

Subsequently, these meanflow calculations were utilized in both linear stability and direct numerical simulations of the flap-edge flow field to calculate unsteady surface pressures and farfield acoustic spectra. Accurate calculations were critical in obtaining not only noise source characteristics, but shear layer correction data as well. Techniques utilized in these investigations as well as brief overviews of results will be given.
\end{abstract}

\section{Introduction}

The importance of reducing subsonic approach airframe noise has now become apparent to the international community. ${ }^{1}$ Civil air traffic continues to increase as does pressure from the public to control the resulting increase in landing noise which is particularly annoying to those living in close proximity to airports. It is clear that noise reduction technology is critical to the future development and operation of the world's air transportation system.

\footnotetext{
1 This paper is declared a work of the U.S. Government and is not subject to copyright protection in the United States.
}

In response to the need for acceleration and augmentation in key subsonic technologies, NASA initiated the Advanced Subsonic Technology (AST) Program in Fiscal Year (FY) 1992. The NASA Noise Reduction Program began in FY 1994 under the AST Program and incorporated several key high payoff areas critical to the development of a new generation of environmentally compatible aircraft. ${ }^{1}$ The Noise Reduction Program established a goal of a 10dB community noise impact reduction relative to 1992 subsonic transport technology. The goal will be achieved by combined noise reduction improvements in the engine system, the aircraft and its operations. In FY95 the Noise Reduction Program began an intense effort in airframe noise source reduction and this research will be the focus of this report. NASA also has a newly instituted base research program in airframe noise under the Advanced Concepts to Test (ASCOT) and Futuristic Airframe Concepts and Technologies (FACT) Programs in FY98. All these efforts will be critical in achieving the environmental goals set by NASA Administrator Daniel S. Goldin "to reduce the perceived noise levels of future aircraft by a factor of two from today's subsonic aircraft and by a factor of four within 20 years."

NASA's airframe noise effort under the AST Noise Reduction Program involves partners in industry and academia. NASA Langley Research Center's (LaRC) role is to determine fundamental noise source mechanisms by relating sound generation mechanisms to fundamental fluid mechanics. This is a critical need of our industry partners, since past predictions are largely based on empirical data that no longer suit the newer classes of aircraft. LaRC couples its building block experiments and computations to full configuration and large-scale tests carried out at NASA Ames Research Center (ARC) as well as flight data made available by Boeing. In addition, Lockheed Martin is a third partner performing computational aeroacoustics for experimentally defined sources. The 
on the flap-edge noise source which began in 1995. Our objective is to obtain direct information regarding the actual noise generation mechanisms responsible for the spectrum produced by the flap-edge flowfield.

\section{Technical Approach: Components}

Since the mid-70's researchers have found that the primary airframe noise sources emanate from the highlift system and undercarriage of subsonic aircraft. ${ }^{3,4}$ Depending on the type of aircraft, the dominant source vacillates between flap, slat and gear. Since none of these components are designed with aeroacoustics in mind, it is no wonder that their very structure gives rise to noise. In an effort to begin a thorough study of these complicated sources, a systematic investigation was initiated at NASA LaRC, to look at the details of the meanflow surrounding these aircraft structures. Components (i.e., flap and slat) were first tested in isolation with simple models that gave rise to the same dominant acoustic source maps that were seen in full configuration tests and at larger scale. The models investigated were 3-element, unswept, partial span flap configurations. In both the flap and slat flowfields large-scale coherent structures were seen to dominate the flowfield. Details of these complicated vortical and separated flow systems were investigated with advanced experimental and computational tools; giving rise to finer scale studies of the fluid mechanics and acoustics which could potentially play a role in airframe noise generation. The work to be discussed focuses on the flap-edge noise source.

Following detailed investigations of the steady flowfield both experimentally (on- and off-surface data) ${ }^{5}$ and computationally (Reynolds-Averaged Navier-Stokes), ${ }^{6,7}$ flowfield fluctuations were measured using hot wire and hot films. The steady flowfield was also investigated for sources of unsteadiness using numerical simulations with the RANS base flow ${ }^{8}$ as well as linear stability which pinpointed dominant frequency ranges of unstable flow disturbances. ${ }^{9}$ These efforts guided the correlation of acoustic measurements with proposed noise sources. Surface unsteady pressure measurements helped characterize the signature of the source followed by microphone array technology which obtained both quantitative spectra and farfield directivity. ${ }^{10,11}$

Maintaining a simplified model, tests were performed at flight Reynolds number to determine the robustness of both the fluid mechanics and acoustics as this important parameter was increased. These tests, performed in NASA LaRC's Low Turbulence Pressure Tunnel (LTPT) ${ }^{12}$ also enabled for a range of velocity sweeps at constant Reynolds number so that reliable sources were tried so that cause and effect could be both understood and substantiated.

\section{Experimental Studies}

Experimental investigations guided the more detailed computational work covered in a subsequent section. The most in-depth studies of both the fluid mechanics and acoustics of the flap-edge flowfield was conducted in the LaRC Quiet Flow Facility (QFF). The airfoil was a NACA $63_{2}-215$ Mod B wing (16-inch chord, 36 inch span) with a $30 \%$ chord half-span Fowler flap. This geometry was also tested in the NASA Ames 7x10 wind tunnel, ${ }^{13}$ a non-anechoic facility, which utilized the model at about twice the size.

Initial investigations of the flap-edge flowfield involved laser light sheets and oil flows. ${ }^{14}$ Laser light sheet images revealed a dominant vortex in the vicinity of the flap-edge. However, a short video constructed from the laser light sheet data did not reveal significant vibration of this structure (a noise generation mechanism originally conjectured). The signature of the vortex track on the flap edge surface was captured by oil flow applications on the pressure and suction side of the flap and main element. Fig. 1. shows these oil flow patterns. ${ }^{5,14}$ The curved streamlines seen in both the suction and pressure surfaces give evidence of the flapedge vortex. In addition, a smaller focus of streamlines on the flap side edge much closer to its trailing edge indicates the presence of a second, smaller vortex.

Further substantiation of the double vortex system are clearly seen in 5-hole probe studies performed in the QFF. ${ }^{7}$ The measurements shown in Fig. 2 are normal planes of vorticity on the flap edge taken from these studies. The dual vortex system is clearly seen. The downstream planes indicate one dominant vortex resulting from merging of both vortices. Note that at the trailing edge, the vortex is far removed from the flap surface.

Acoustic maps of high intensity noise on the flap side-edge closely mirror the fluid mechanics of the flow. Two microphone array systems were developed at NASA LaRC to quantify these results. ${ }^{11}$ A large aperture array using 35 microphones was constructed to obtain high resolution noise maps. This array possesses a maximum diagonal aperture size of 34 inches. A unique logarithmic spiral layout design was chosen for the targeted frequency range of $2-30 \mathrm{kHz}$. In addition, a small aperture array, constructed to obtain spectra and directivity, complemented the larger design. This small array possesses 33 microphones with a maximum diagonal aperture size of 7.76 inches. It was easily moved in both azimuth and elevation about the model mounted in the QFF. Custom microphone shading 
$40 \mathrm{kHz}$ with an overall targeted frequency range for the array of 5-60 kHz. Both of these arrays were used with the NACA $63_{2}-215$ Mod B wing model described above. In Fig. 3., source localization maps from the large aperture array chart the progression of the hot spot for frequencies from 5 to $20 \mathrm{kHz} .{ }^{11}$ At the higher frequencies, this hot spot is localized on the edge of the flap. This is consistent with the primary vortex grazing the edge of the flap. As the frequency decreases the hot spot moves downstream and inboard, while the merged vortex system comes over the edge impinging on the upper surface. Recently reported noise reduction schemes by NASA Ames ${ }^{15}$ indicate that flap-edge fences, which increase distance between the vortex system and the surface, can achieve noise reduction.

A test conducted in the LaRC LTPT on a second model of a part-span flap was co-investigated by NASA's High-Lift Program element and LaRC's Airframe Noise team. This model, known as the Energy Efficient Transport (EET) model ${ }^{16}$ has a vastly different cove design as well as camber on a flap optimized for high-lift. The LTPT test allowed Reynolds numbers to range from 3.6 to 19 million based on chord. A further advantage of this pressure tunnel was the range of velocities achievable at constant Reynolds number, an important aspect for obtaining accurate scaling laws. This was the first test performed with an acoustic array at these high Reynolds number conditions. Array development ${ }^{17}$ and data acquisition was performed by the Boeing Commercial Airplane Company. Although the primary acoustic sources present in the QFF/7x10 experiments appeared in the EET study, an additional hot spot at low frequency was also seen off the trailing edge of the flap. The acoustic image map indicating this additional source is given in Fig. 4b, in contrast with the map of the previously observed source shown in Fig. 4a. Details of the flowfield that corroborate this finding are discussed in the next section.

In virtually all studies conducted an abrupt rise in noise intensity occurred following an increase in flap deflection. $^{10,18}$ It was found both experiementally ${ }^{5}$ and computationally ${ }^{7}$ that both flap deflections also induced vortex bursting on the flap-edge system. The correlation of this event with noise is ongoing.

\section{Computational Studies}

An intensive investigation of the fluid dynamics associated with the high-lift system began in FY 1995. Mirroring the discussion above, these studies aimed at elucidating the flowfield surrounding the part span flap.

Initially, computations focused on the model tested in the QFF and 7x10 facilities. Validation of a Reynolds-Averaged Navier-Stokes solution is given in obtained in the $7 \times 10$ experiment compared against several of these computations. Most of the studies were performed using CFL3D on a structured mesh utilizing the Spalart-Allmaras turbulence model. An excellent comparison of Pressure Sensitive Paint (PSP) and computations of the vortex signature on the suction surface of the flap are given in Fig. 6. ${ }^{7}$ PSP was the key measurement technique for obtaining details on the edge of the flap, and was used to validate the computational findings. However, oil flow methods were utilized on the flap edge ${ }^{14}$ surface as well. Figure 7 compares oil flows and computational streamlines for a highly loaded flap setting. Note the accumulation of oil at approximately $2 / 3$ chord. The separation and attachment lines abruptly end in this focal point. The computations clearly show that downstream of this point the flow reverses and moves upstream. ${ }^{7}$ The most dramatic proof of the vortex bursting which gave rise to this focal point, occurred when Particle Image Velocimetry (PIV) was performed in the LaRC Basic Aerodynamic Research Tunnel. ${ }^{21}$ Remarkable agreement with the calculations was obtained. Fig. 8 depicts streamwise velocity plots from RANS and PIV. Note the large region of reversed flow where the vortex lifts off the surface while undergoing bursting.

The above studies led to a series of systematic investigations utilizing the RANS as the model meanflow for both linearized stability studies ${ }^{9}$ and temporal simulations. ${ }^{8,22}$ The frequency ranges, calculated from the linear stability analysis, exhibited the highest growth rates; the numerical simulations indicated that these frequency ranges also had the most explosive growth. The latter calculations were interfaced with a Lighthill Acoustic Analogy, obtaining the first noise calculations for this complicated geometry. ${ }^{22}$ The hot spots obtained in the QFF seemed to be explainable in terms of both cylindrical shear layer and vortical instabilities as discussed in references 8,9 and 22. Fig. 9 shows clearly the strong disturbance vorticity present in the shear layer that rolls into the flap-edge vortex. The results were calculated from a temporal DNS utilizing a normal cut of the part-span flap RANS solution at approximately $50 \%$ chord. The broadband nature of this instability becomes apparent, as this disturbance remains significant from 5 to 30 $\mathrm{kHz}$. However, at the lower frequencies the vortex instability appears to be stronger than that of the shear layer. Recall from Fig. 3 that the $5 \mathrm{kHz}$ noise map indicates the maximum in intensity occurring inboard of the flap-edge at the location where the vortex moves rapidly over the edge and onto the suction surface (Fig. 2 ). The higher frequency noise maps in this figure indicate maxima more along the edge where the shearlayer instabilities should be dominant; this is confirmed 
reader is referred to references 8 and 22 for more detail.

As alluded to in the previous section another source appeared during acoustic measurements on a second part-span flap model, the EET. Recall Fig. 4, which indicates a high noise region just off the trailing edge of the flap at low frequency. When the RANS solution was performed for the EET geometry, a dramatic difference in vortex trajectory was discovered, relative to that seen in the $63_{2}-215$ flowfield. Fig. 10a indicates planes of vorticity along the flap-edge of this model. If one contrasts these cuts with that of the $63_{2}-$ 215 flowfield (Fig. 10b) the vortex is seen to stay in close proximity to the edge surface of the flap down to the trailing edge for the EET flowfield. In contrast, the $63_{2}-215$ model flowfield has the vortex abruptly leaving the surface by around $60 \%$ chord. By the time the flow reaches the trailing edge in that case, the vortex is far-removed from any solid surfaces. It is believed that fluctuations in the vortex, being much nearer to the flap surface and trailing edge for the EET model, potentially give rise to the additional hot spot of Fig. 4. Again, the acoustic signatures closely mirror the findings of the fluid mechanics.

\section{Conclusions}

Fundamental studies of airframe noise sources for subsonic aircraft are being conducted at NASA Langley Research Center. The work presented deals with the noise generated by the flap-edge flowfield. A series of detailed experimental and computational building block studies were described corroborating several key noise source mechanisms associated with this important component. Coordination of acoustic source maps with Reynolds- Averaged Navier-Stokes, linear stability analysis, and numerical simulations gave rise to plausible noise generation mechanisms stemming from both a cylindrical shear layer and a primary vortex structure. Newly designed array technology allowed for high Reynolds number testing in a hard wall facility, along with more detailed quantitative acoustic measurements in an anechoic chamber. Calculations of highly accurate mean flows as well as unsteady flow characteristics gave excellent agreement with both fluid mechanics and acoustic experiments of several part-span flap models. This work is part of a larger effort in the NASA AST Noise Reduction Program, which includes large scale testing at NASA Ames and Boeing Commercial Airplane Company as well as computational aeroacoustics performed at Lockheed Martin.

\section{Acknowledgements}

The author would like to thank William Willshire, Program Manager of the Noise Reduction Program for responsible for results given: Dr. C. Streett, Dr. T. Brooks, Dr. W. Humphreys, Dr. M.Khorrami, Professor Geoffrey Lilley, Dr. C. Gerhold, Dr. D. Lockard, Dr. K.Meadows, Dr. R. Radeztsky, Dr. W. Hunter, Dr. B. Singer, Dr. M.Takallu, M. Sanetrik Dr. W. Anderson, J.Underbrink, R. Stoker, and G. Neubert.

\section{References}

1. Willshire, W.L. and Stephens, D.G.: "Aircraft Noise Technology for the 21st Century", NOISECON 98.

2. Goldin, Daniel S.: "Turning Goals into Reality," presented at World Aviation Congress, Los Angeles, CA, Oct. 15, 1997.

3. Crighton, D.G.: "Airframe Noise in Aeronautics of Flight Vehicles: Theory and Practice," Vol. 1; Noise Sources, NASA RP 1258, pp. 391-447, 1991.

4. Sen, R.: "A Study of Unsteady Fields Near Leading-edge Slats,” AIAA 97-1696, 1997.

5. Radezrsky, R.H., Singer, B.A., and Khorrami, M.R.: "Detailed Measurements of a Flap SideEdge Flow Field," AIAA 98-0700, 1998.

6. Takallu, M.A., and Laflin, K.R.: "ReynoldsAveraged Navier-Stokes Simulations of Two Partial-Span Flap Wing Experiments," AIAA 980701, 1998.

7. Khorrami, M.R., Singer, B.A. and Radeztsky, R.H.: "Reynolds Averaged Navier-Stokes Computations of a Flap Side-Edge Flow Field," AIAA 98-0768, 1998.

8. Streett, C.L.: Numerical Simulation of Fluctuations Leading to Noise in a Flap-Edge Flowfield," AIAA 98-0628, 1998.

9. Khorrami, M.R., and Singer, B.A.: "Stability Analysis for Noise-Source Modeling of a PartSpan Flap," AIAA 98-2225, 1998.

10. Meadows, K.R., Brooks, T.F., Gerhold, C.H., Humphreys, W.M . and Hunter, W.W.: Acoustic and Unsteady Surface Pressure Measurements of a Main Element-Flap Configuration," AIAA-971595.

11. Humphreys, W.M., Brooks, T.F., Hunter, W.W., and Meadows, K.R.: "Design and Use of Microphone Directional Arrays for Aeroacoustic Measurements," AIAA 98-0471, 1998.

12. McGhee, R.J., Beasley, W.D., and Foster, J. M.: "Recent Modifications and Calibration of the Langley Low-Turbulence-Pressure-Tunnel," NASA TP-2328, 1984.

13. Storms, B.L., Takahashi, T.T., and Ross, J.C.: "Aerodynamic Influence of a Finite-Span Flap on a Simple Wing," SAE Paper 951977, 1995. 
Langley Research Center.

15. Horne, C.F., Hayes, J., and Ross, J.C.:

"Measurements of Unsteady Pressure Fluctuations on the Surface of an Unswept Multi-Element Airfoil," AIAA 97-1645, 1997.

16. Morgan, H.L., Jr.: "Model Geometry Description and Pressure Distribution Data from Tests of EET High-Lift Research Model Equipped with FullSpan Slat and Part-Span Flaps," NASA TM-80048, 1979.

17. Underbrink, J.R. and Dougherty, R.P.: “Array Design for Non-intrusive Measurements of Noise Sources," NOISECON 96, 1996.

18. Hayes, J.A., Horne, C.W., and Bent, P.H.: "Airframe Noise Characteristics of a $4.7 \%$ Scale DC-10 Model," AIAA-97-1594-CP, 1997.

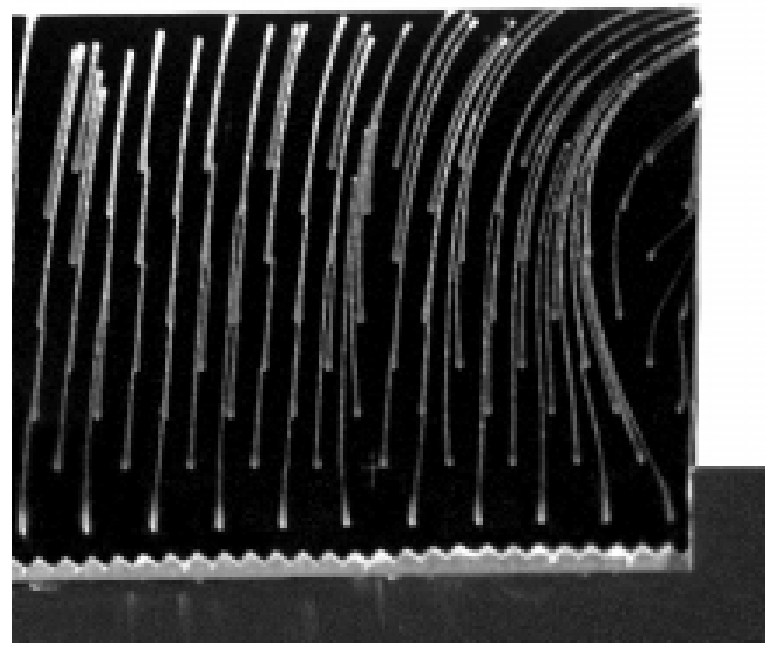

a) suction surface
"Implicit/Multigrid Algorithms for Incompressible Turbulent Flows on Unstructured Grids," Journal of Computational Physics, vol. 128, pp. 391-408, 1996.

20. Mavriplis, D.J., and Venkatakrishnan, V.: "A Unified Solver for Navier-Stokes Equations on Mixed Element Meshes," International Journal for Computational Fluid Dynamics," vol. 8, pp. 247263, 1997.

21. PIV measurements performed in the NASA LaRC BART facility by L. Jenkins, P. Yao, and K. Paschal.

22. Streett, C.L.: "Numerical Simulation of a FlapEdge Flowfield," AIAA 98-2226, 1998.

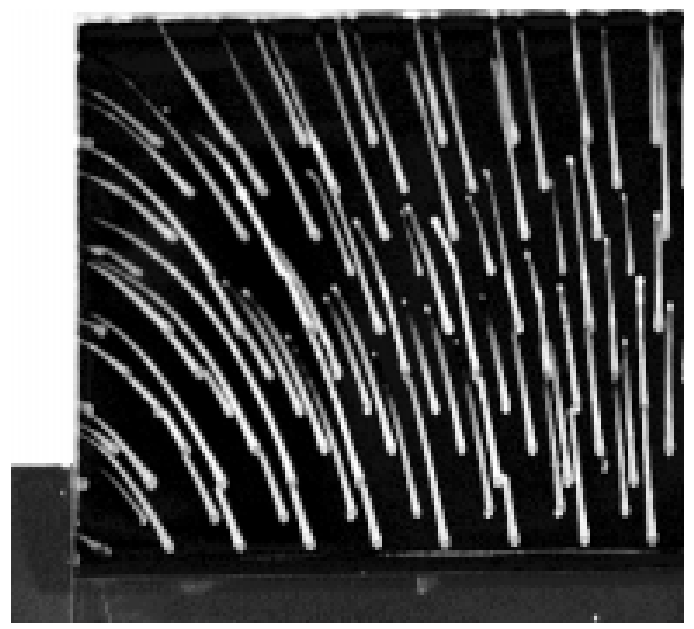

b) pressure surface

Figure 1. Oil flow patterns on the flap-edge of the $63_{2}-215 \operatorname{Mod} B$ wing.

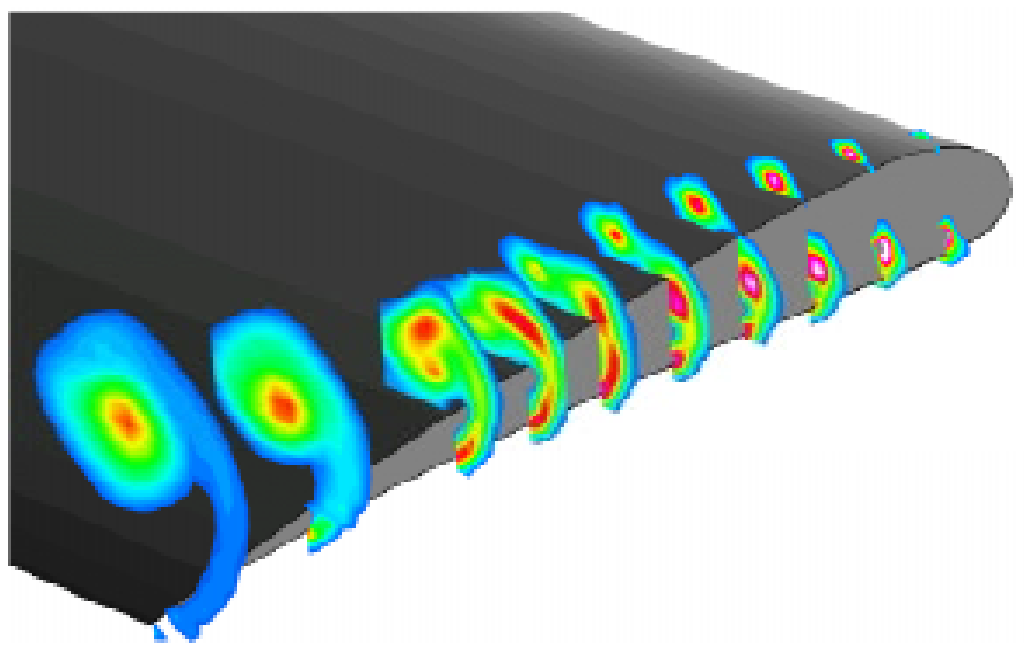



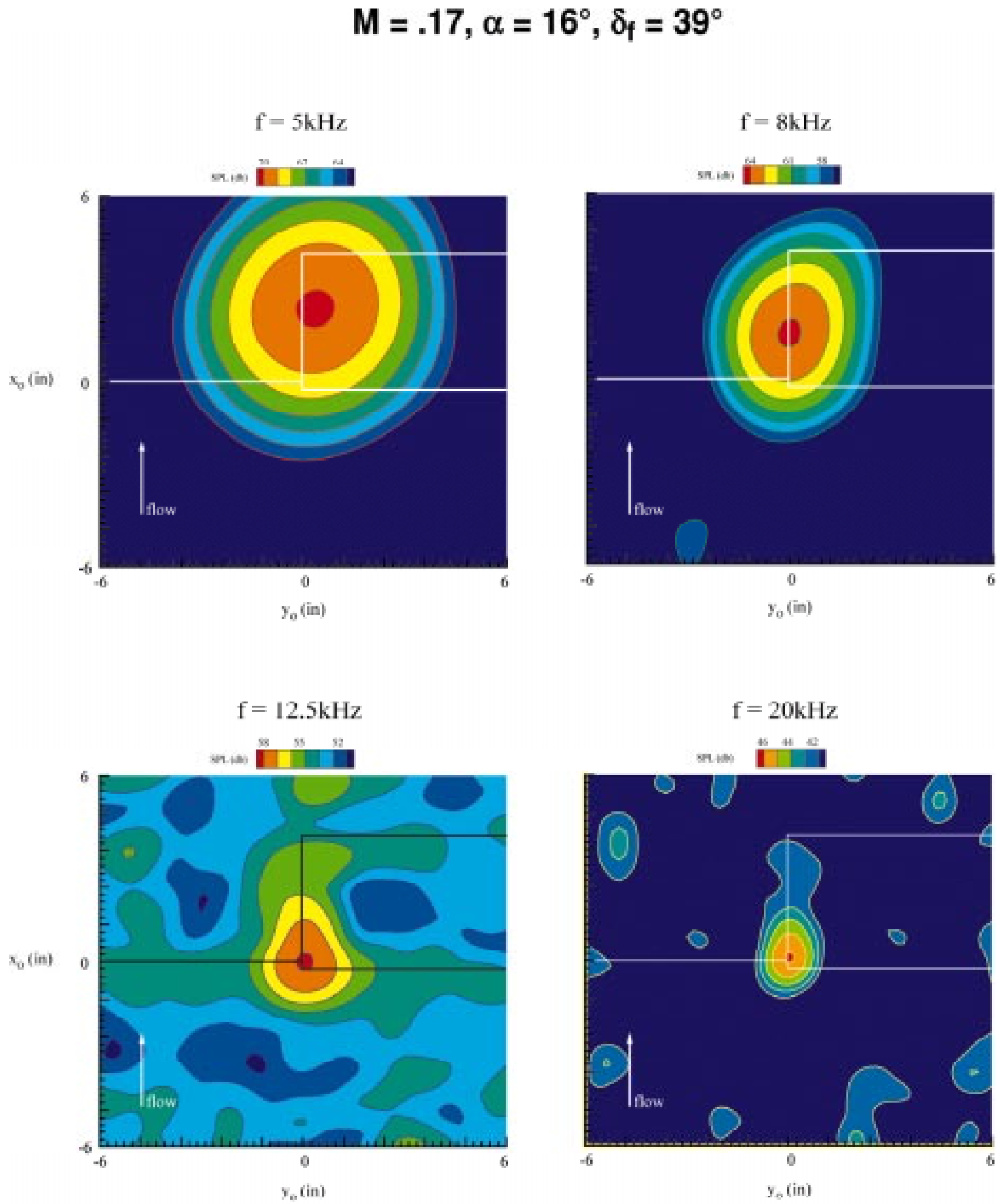

Figure 3. Sound source localization maps from acoustic array measurements in the Quiet Flow Facility. 


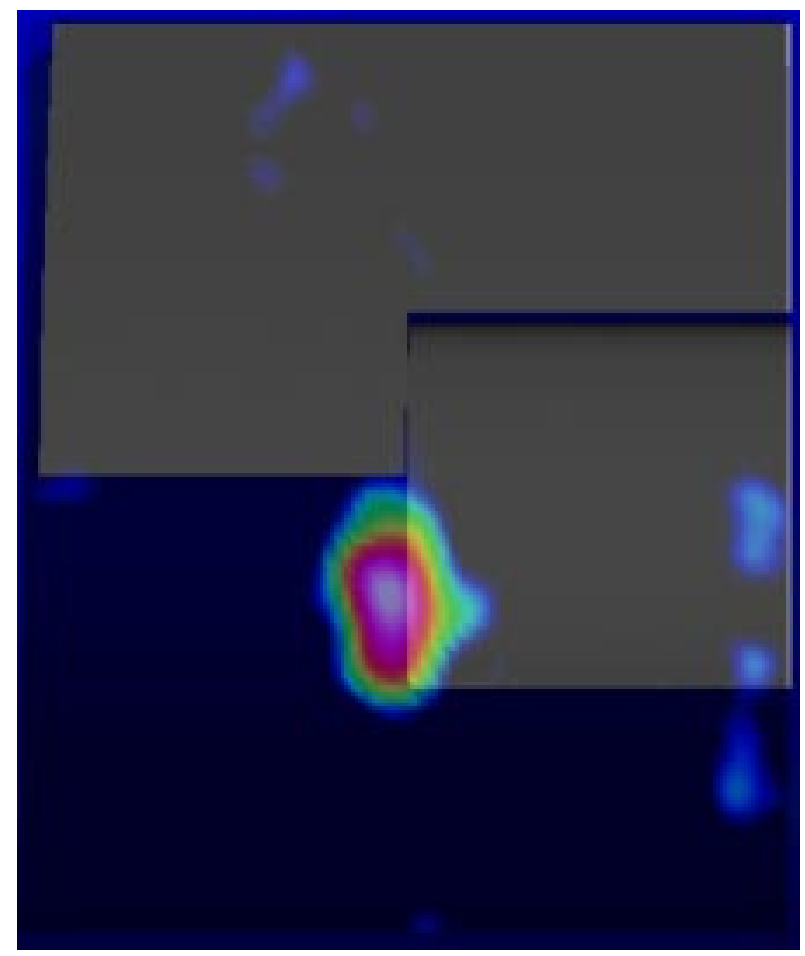

a) side-edge source

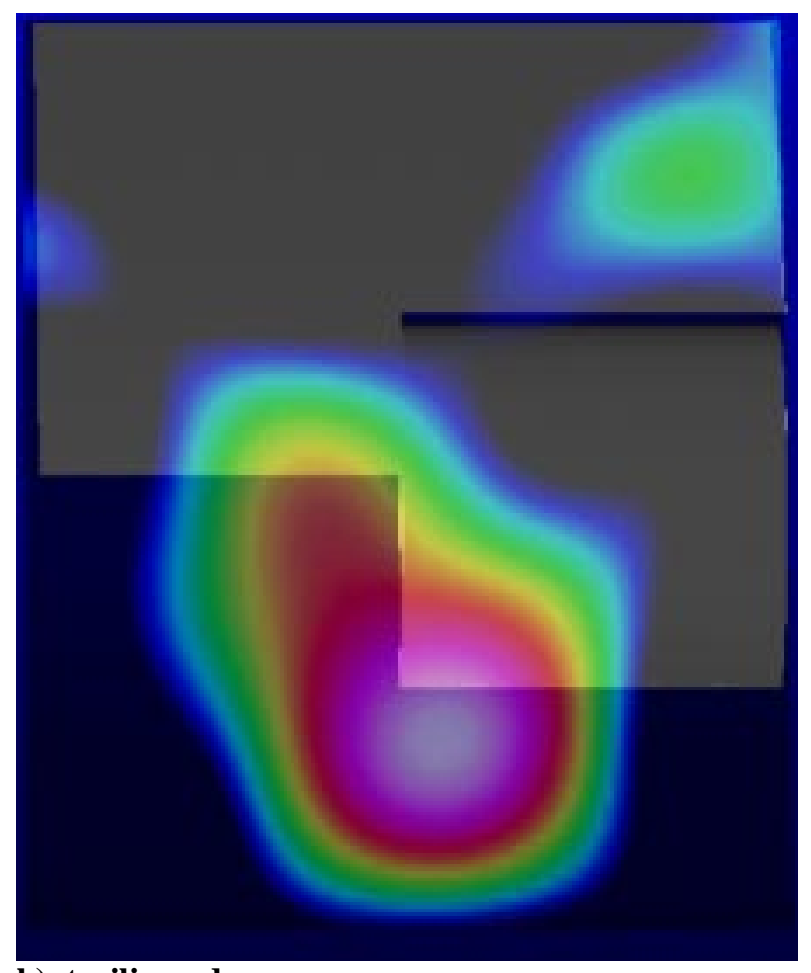

b) trailing-edge source

Figure 4. Sound source localization map from EET part span model in the Low Turbulence Pressure Tunnel.
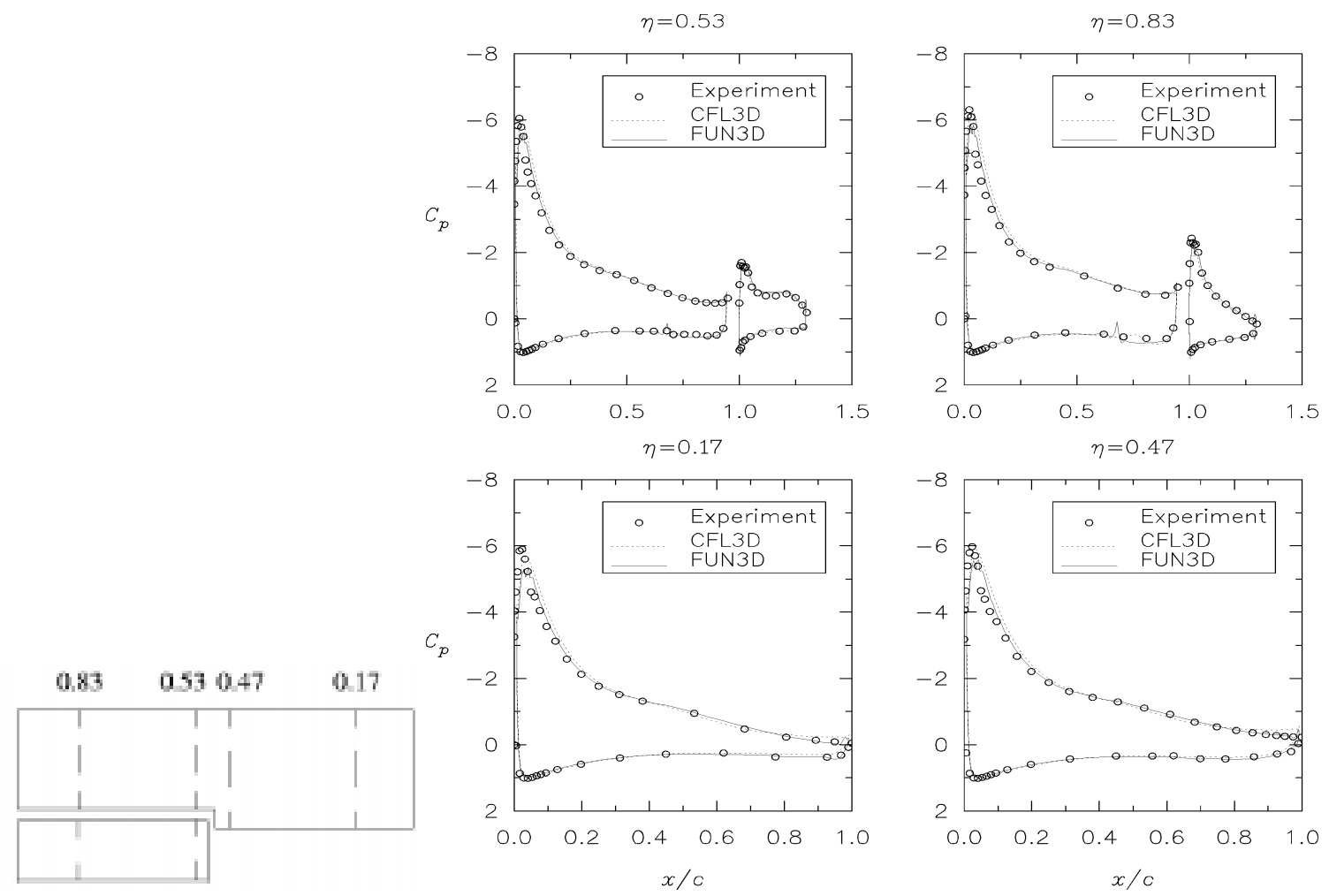

Figure 5. Experimental and computational Cp profiles from the 7x10 flap-edge test: CFL3D - structured, 


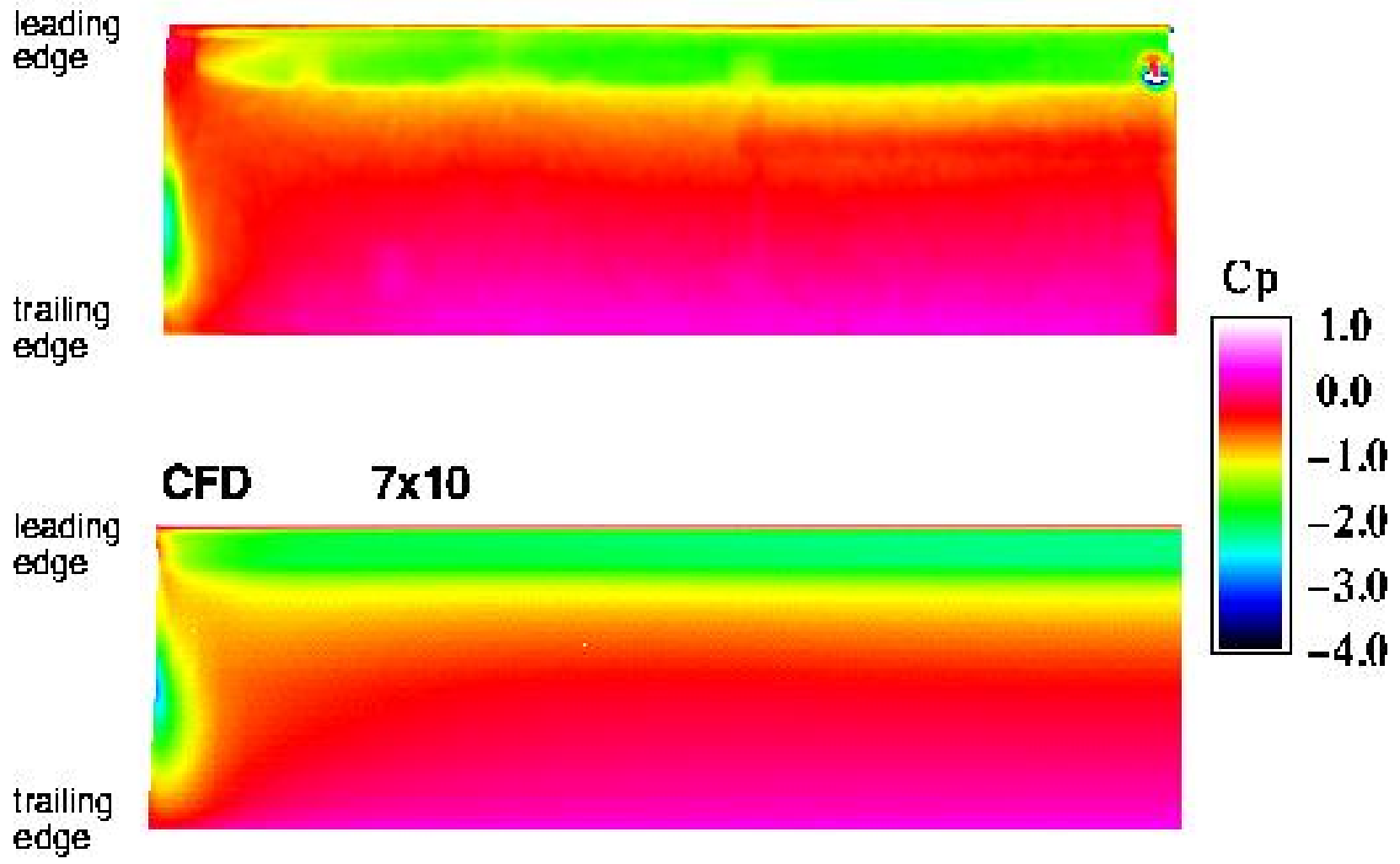

Figure 6. Comparison between computed and pressure sensitive paint measurements taken in the QFF on the suction surface of the flap.

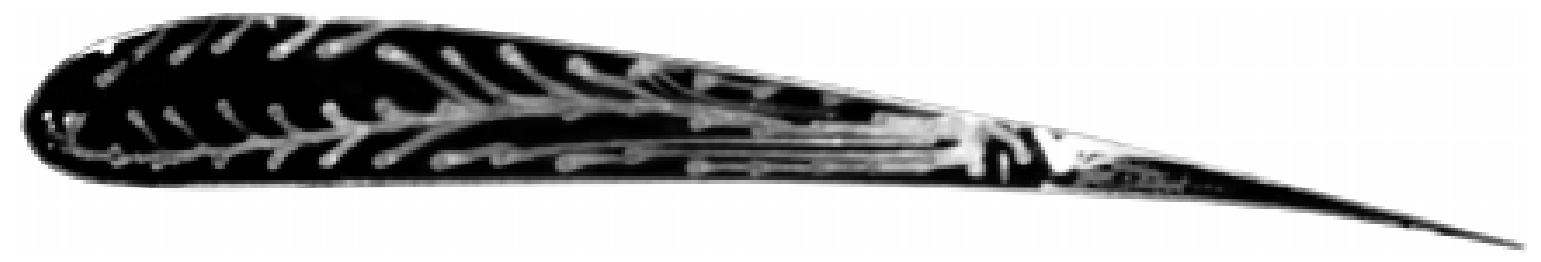

a) experiment - oil flow

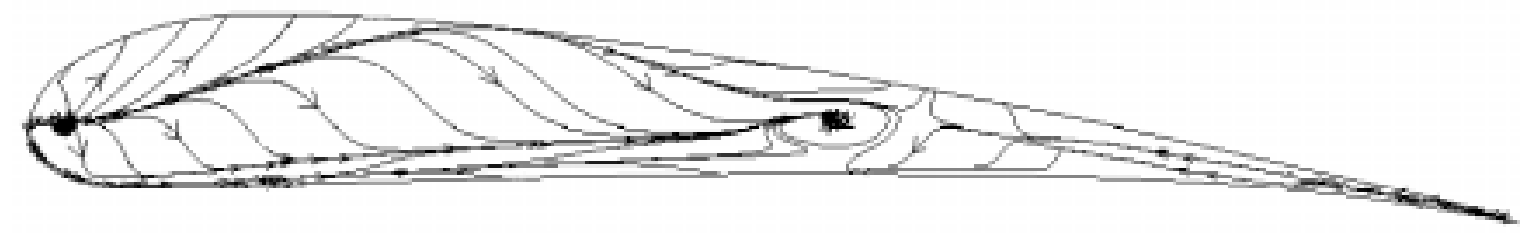

b) CFD - surface streamlines

Figure 7. Flap side-edge surface streamlines indicating focal point for flow reversal at high flap deflection. 


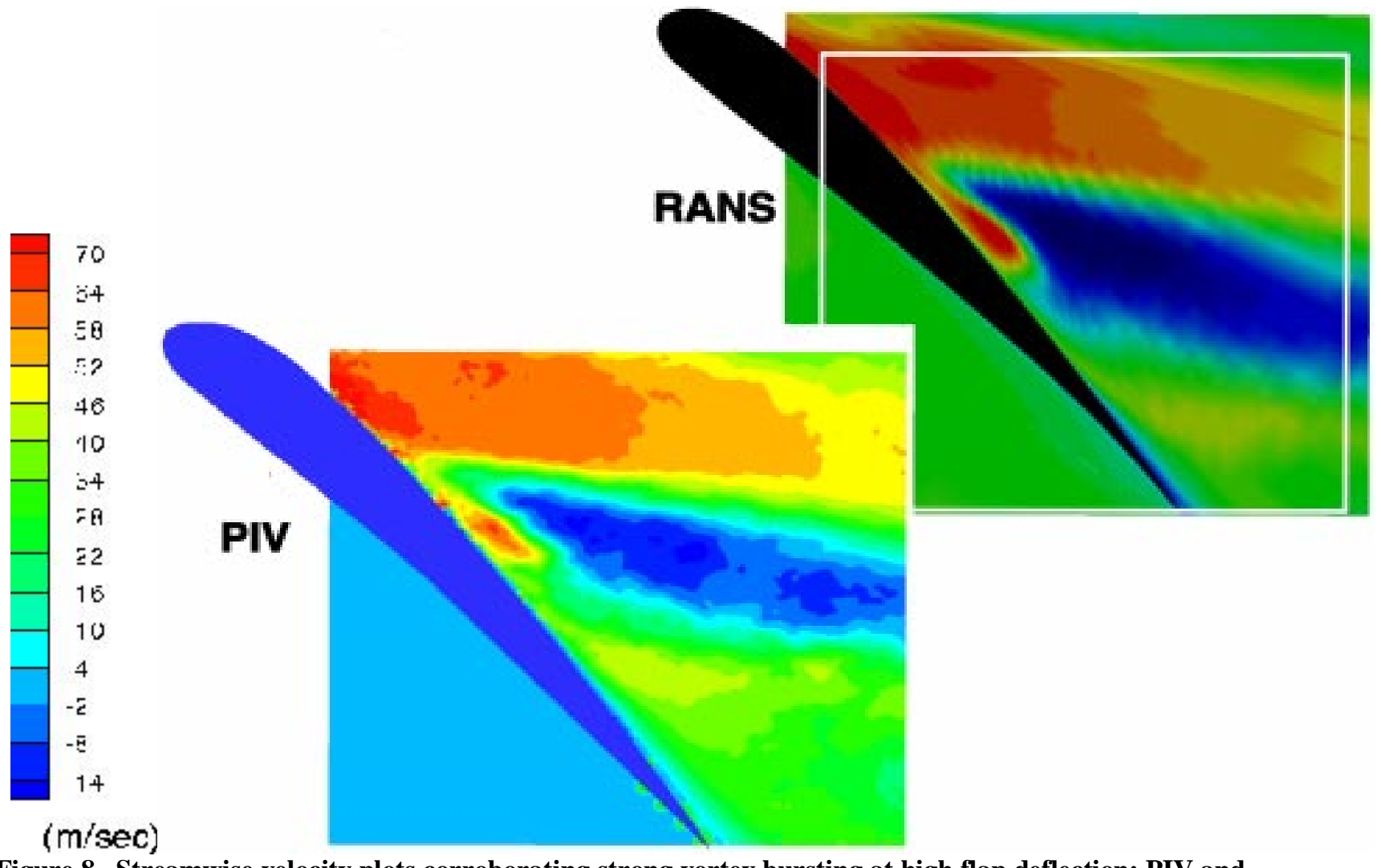

Figure 8. Streamwise velocity plots corroborating strong vortex bursting at high flap deflection: PIV and RANS comparison.
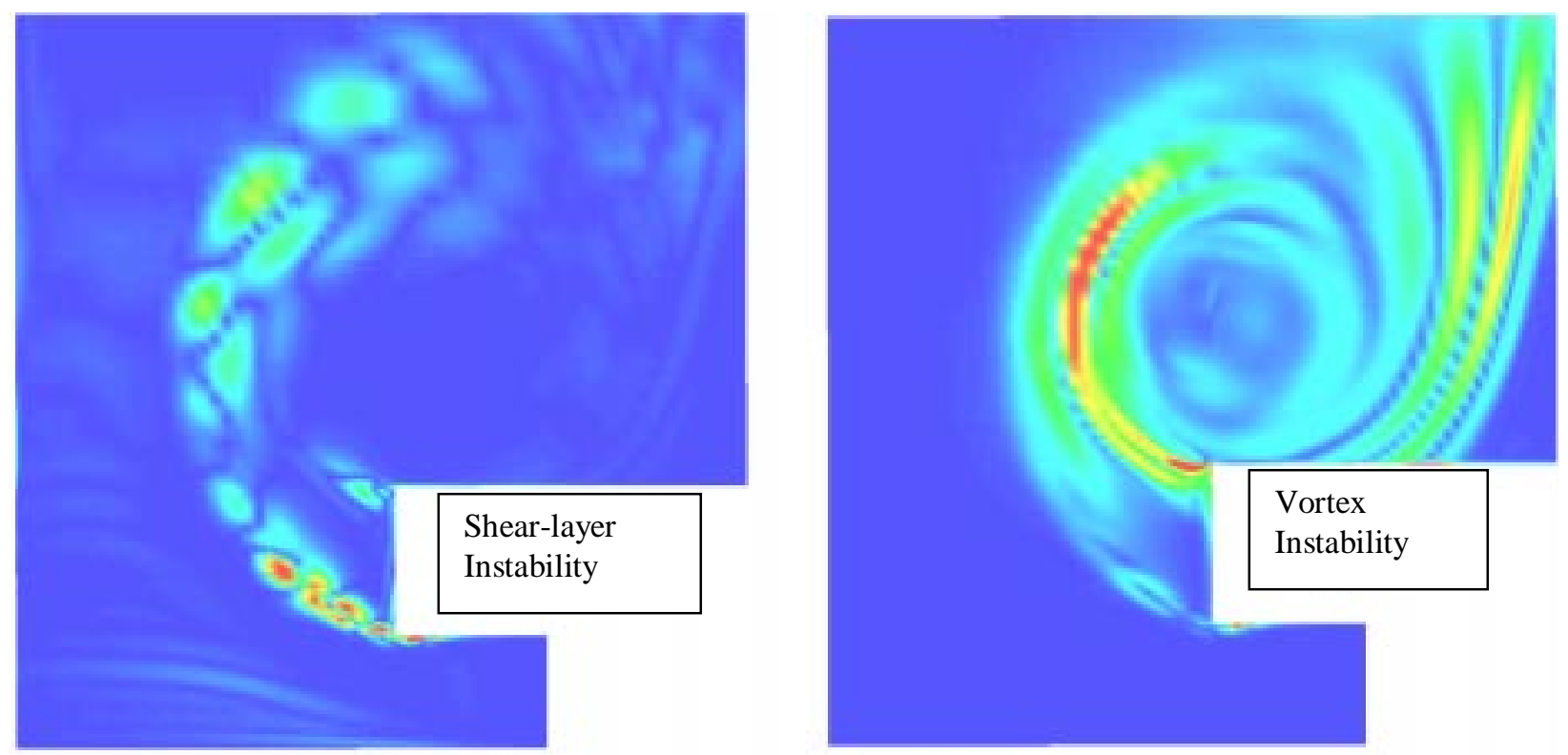

Figure 9. Calculated disturbance vorticity from shear-layer and vortex instabilities, from temporal DNS linearized about a normal cut of a Reynolds Averaged Navier-Stokes solution; 50\% flap chord. 

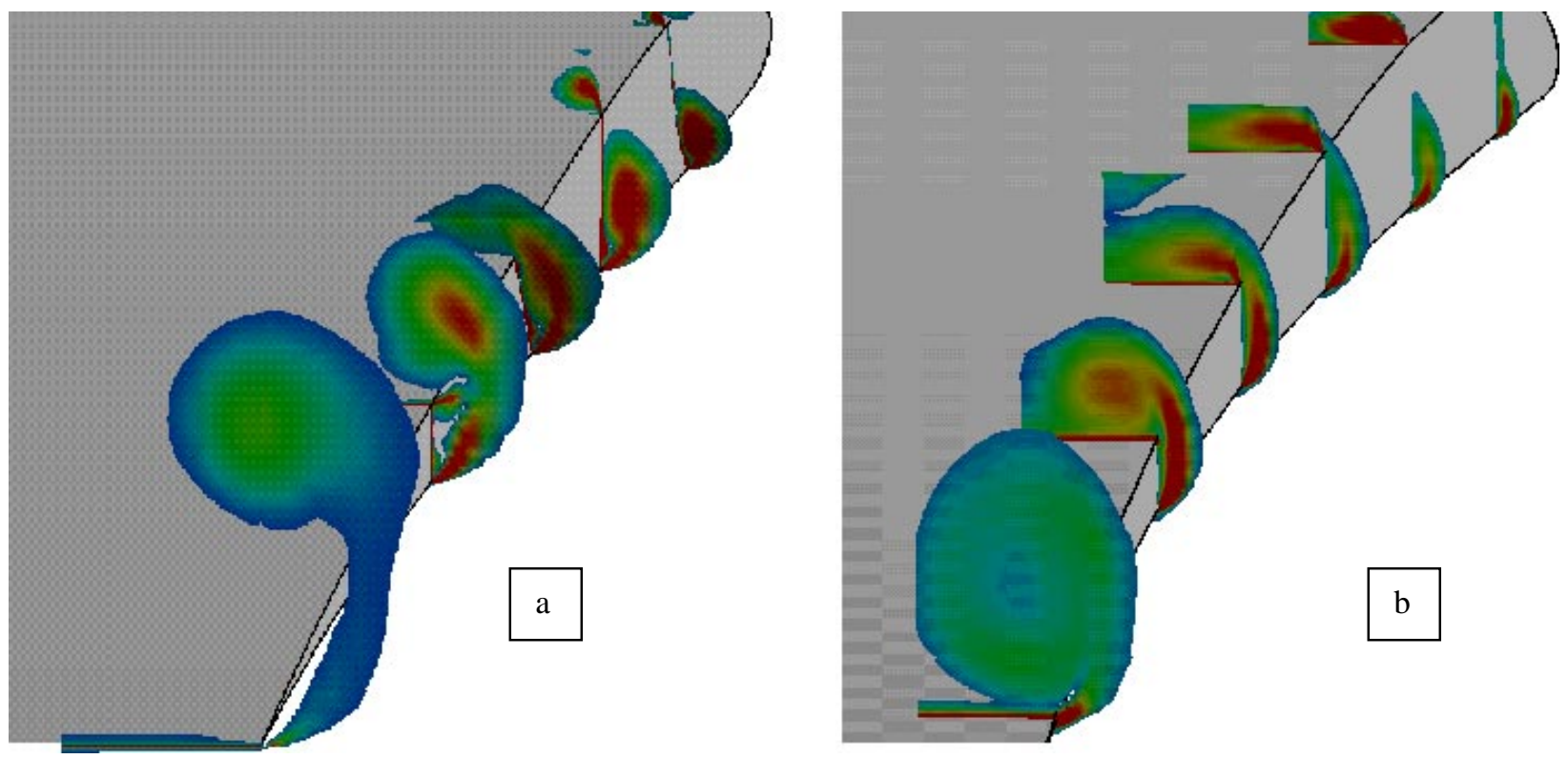

Figure 10. Comparison of streamwise vorticity contours on the flap side edge for two part-span flap models: a) NACA 632-215 (QFF/7x10 model); b) EET (LTPT model) 